University of San Diego

Digital USD

Spring 5-25-2019

\title{
Implementing a Trigger-Based Model for Early Palliative Care Consultation on a Medical Oncology Floor
}

\author{
HSIU JAN CHEN \\ University of San Diego, hchen@sandiego.edu
}

Follow this and additional works at: https://digital.sandiego.edu/dnp

Part of the Nursing Commons

\section{Digital USD Citation}

CHEN, HSIU JAN, "Implementing a Trigger-Based Model for Early Palliative Care Consultation on a Medical Oncology Floor" (2019). Doctor of Nursing Practice Final Manuscripts. 87.

https://digital.sandiego.edu/dnp/87

This Doctor of Nursing Practice Final Manuscript is brought to you for free and open access by the Theses and Dissertations at Digital USD. It has been accepted for inclusion in Doctor of Nursing Practice Final Manuscripts by an authorized administrator of Digital USD. For more information, please contact digital@sandiego.edu. 
Implementing a Trigger-Based Model for Early Palliative Care Consultation on a Medical Oncology Floor

Hsiu Jan (Leora) Chen

University of San Diego 
Implementing a Trigger-Based Model for Early Palliative Care Consultation on an Oncology Medicine Floor

According to National Palliative Care Registry (2017), cancer has been ranked the primary diagnosis for palliative care consultation (26\%), followed by cardiac (15\%), pulmonary (14\%), neurological (9\%), and infectious (7\%) diagnoses. The sequelae associated with cancer metastases have been well documented (Bakitas, Tosteson, \& Li, 2015). Not only do the complex conditions and accompanying comorbidities impact the patients' and their caregivers' quality of life, but they also lead to significant medical costs; a total of $\$ 80.2$ billion was spent on cancer treatment in outpatient and inpatient setting in 2015 (American Cancer Society [ACS], 2017).

The World Health Organization (WHO) describes palliative care (PC) service as valuable support and care for the individual and family need in physical and psychosocial aspects. Many established studies also reveal that patients report satisfied pain and symptom management (e.g., nausea/vomiting, dyspnea, constipation, anxiety, delirium), higher quality of life score when receiving PC in their care. Also, American Society of Clinical Oncology (ASCO) voices the addition of PC consultation to standard oncology medicine treatment for patients with advanced cancer (Bakitas et al., 2015; Ferrell et al., 2016; Parikh, Kirch, Smith, \& Temel, 2013).

\section{Description of the Clinical Problems}

Despite existing support for positive patient care outcomes through early PC consultation, a systematic study shows that $84 \%$ of the providers referred their patient to palliative care at a very late stage for uncontrolled symptom management (Wentlandt $t$ al., 2012). Leading barriers include insufficiency of training, the inadequacy of recognized referral criteria, and referral protocol. Moreover, a delayed PC referral also prolongs patient suffering from symptom distress 
and excessive laboratory work in addition to unnecessary intensive care unit admission (Wentlandt et al., 2012).

A similar clinical problem of the lack of recognized referral criteria and lack of a referral protocol in early PC consultation initiation arose in a newly opened, 12-bed medical oncology floor of an urban, 364-bed academic medical center. The PC consultation model is available to patients in the traditional way where the hospitalist orders a PC consult based on his or her assessment and evaluation of the patient. Given the lack of standard PC referral criteria, it takes more than five days for patients who admitted to this floor receiving PC consultation for various unmet PC needs.

Furthermore, the patients admitted to this medical oncology floor have a higher, unmet PC need when using a PC checklist in recognizing potential candidates compared to actual palliative care written orders by the hospitalists (19\% vs. 8\%). This 3-week retrospective chart audit was performed by adopting a PC trigger tool(see Figure 2)from University of California San Diego (UCSD) Health's Intensive Care Unit (ICU) in reviewing the patient charts from May to June 2017. The chart audit compared the patients who received a traditional PC consultation after it was ordered by their hospitalists and on patients who did not have PC consult but were screened by UCSD ICU PC trigger tool. The result showed that $80 \%$ of patients required aggressive cancer-related pain and symptom management, $66 \%$ of patients needed a goal of care discussion, $52 \%$ of patients had a foreseeable decrease in quality of life (e.g., malignant pleural effusion, venting gastronomy tube, decline of mobility) when following the PC trigger indications. These findings indicated that described the goals of care and proactive symptom management were not adequately addressed; furthermore, chances of prolonged suffering and length of hospital stay may result in more physical, emotional, and financial distress in patients 
and their families. In short, PC services specializing in assisting the timely goal of care discussion and aggressive cancer-related symptom management are urgently needed.

\section{Intended Improvement and Study Question}

In improving a timely PC consultation, a evidence-based practice (EBP) pilot study was initiated based on the PICO (Problem/Patient/Population, Intervention/Indicator, Comparison, Outcome) on how would utilization of a PC trigger tool in early palliative care consultation impact patient outcome on the length of stay, cancer-related symptom management, and goal of care discussion initiation.

This project used the Iowa evidence-based practice (EBP) model to guide the project implementation process. There are two reasons for selecting Iowa model in this project. First, the Iowa model provides a comprehensive diagram which presents less confusion for the project team during the project developing process. Also, it assists the team in identifying the most priority of the clinical problem at the beginning of the stage. Second, this model has been applied for both clinical or research problem solution, with a less restriction, Iowa EBP model are commonly used in different patient populations, EBP projects, and units. Therefore, the Iowa model endowed with an ideal guide for this pilot study implementation. (Buckwalter et al., 2017; Melnyk \& Fineout-Overholt, 2014).

\section{Proposed Evidence-Based Solutions}

A literature review of relevant studies on the strategy of early PC consultation implementation, time of PC consultation initiation, consultation criteria, and the outcome was conducted. Keywords such as inpatient early palliative care, palliative care triggers, palliative care indication, a patient with a solid tumor, patient outcome, and early palliative care 
intervention were applied on PubMed, CINAHL, Google Scholar, and Cochrane websites. A total of 17 articles from 2012-2017 met research criteria.

A hierarchy of evidence for the intervention question scale I-VI is exploited for literature praised and critiqued on the preliminary 17 articles (Melnyk \& Fineout-Overholt, 2014). Five articles were selected for this evidence-based practice project including one systematic review, Integration of Palliative Care Into Standard Oncology Care (Ferrell et al., 2016). The other four articles provide Level II evidence as randomized controlled trial (RCT) studies, proposing a favorable patient outcome on early palliative care compared to the usual care in different settings (Bakitas et al., 2015; Groenvold et al., 2017; Zimmermann et al., 2014).

Ferrell et al. (2016) provided appropriate PC consult indications for providers in recognizing the potential candidates: emotional distress, unrelieved cancer-associated symptoms, a lack of understanding of illness and/or poor prognosis, clarification of treatment options and anticipated outcomes, the need to discuss goals of care, assistance with medical decision making, and coordination of care for other care providers. Furthermore, when identifying that a patient has a limited life of 6-24 months, providers should make a proactive PC referral for extra care and support for patient and family (Ferrell et al., 2016).

A couple of RCT results support that patients report better life quality, less symptom intensity, and a higher spiritual-well being through the care by oncological care incorporated with PC service. In Groenvold et al. study (2017), patients with newly diagnosed of stage III to IV received early PC consultation had better nausea and vomiting controlled. Bakitas et al. (2015) pointed out the patients with advanced cancer who received an early PC consultation within three months after the diagnosis showed lower Emergency room visits, lower hospital days and less intensive care unit admissions by the regular telephone follow-up on symptom 
management, coping strategy education, crisis resource access. Significantly, the early group showed a more prolonged survival length of one year compared to the delayed group who received PC consultation three months later.

Using triggers for early PC consultation also studied in various patient setting including Emergency room, oncology inpatient floor, and intensive care unit. A single-blind randomized trial showed Emergency Department physicians using a PC trigger for early PC consultation when advanced cancer patients presented in ED for symptom management, the goal of care discussion, and the result showed a less waiting time to consult of 1.48 days compared to the usual care of 2.9 days. (Kistler, Sean Morrison, Richardson, Ortiz, \& Grudzen, 2015). Another Trigger-based PC consultation in an oncology inpatient setting shows positive outcomes on higher PC consult rate, lower 30-day readmission rate, decreased chemotherapy use in the last two months of a patient's life. In this oncology floor, the PC consultation is automatically activated when a patient with advanced cancer who meets the criteria of the previous admission within 30 days, hospitalization more than seven days with active cancer-related symptoms. (Adelson, Paris, Horton, Hernandez-Tellez, Ricks, 2017). Even in ICU, the PC trigger has been seen as a tool of patient care when a patient's terminal condition meets the points of the transition plan discussion. The trigger may be different by the units, but, all in all, trigger plays a role in lessening subjective assessment to individual physicians instead of promoting an objective assessment in identifying patient unmet need for PC consultation promptly. (Creutzfeldt, Wunsch, Curtis, \& Hua, 2015; Hua, Li, Blinderman, \& Wunsch, 2014)

\section{Methods}

\section{Study Design and Participants}


The EBP pilot study aims to facilitate an early PC consultation on an oncology medicine floor by implementing a PC consultation screening tool, promote less waiting time from admission to initial PC consultation by one day, supporting optimal cancer-related symptom management (pain, nausea, dyspnea, constipation), and decrease of the length of stay by one day by end of November 2018 .

The project proposal was reviewed by the UCSD Institutional Review Board (IRB) and qualified as a quality improvement project (QI) on May 8, 2018. This pilot QI project was implemented on a 12-bed oncology medicine floor at the UCSD Jacobs Medical Center from August 23, 2018, to November 28, 2018. The pilot project included patients age 18 or older with a diagnosis of either solid tumor or blood tumor admitted to the floor during the intervention period. Patients who had a PC service during their hospital stay before the project initiative were excluded from the project.

\section{Instrument}

Given the patient population in this floor are cancer patients, the PC consult checklist (PC triggers) is adapted from the National Comprehensive Cancer Network's (NCCN) 2016 palliative care consultation guidelines (Levy et al., 2016) instead of continuing using the trigger that was created by UCSD ICU. Through consultation and peer reviews with the UCSD director and nurse practitioner of the palliative care service consulting team on the trigger list formulation, a total seven prominent PC consultation indicators were selected for the PC trigger as guidance. The indicators are: request from a patient/family/caregiver, need for clarification of treatment goals, severe constipation that does not respond to current bowel regimen, delirium, dyspnea, severe pain that does not respond to current pain management regimen, and refractory nausea/vomiting that does not respond to current treatment. (see Figure 1). 
Measurement instrument. The self-reporting numeric rating scale (NRS) was chosen as a pre-post pain intervention tool because it has been used with oncological patients and is easy to apply. The numeric rating scale comprises 11 points to represent pain intensity. A score of 0 means patient self-report pain is no pain; a score of 1-3 means mild pain; a score of 4-6 means moderate pain; and a score of 7-10 means severe pain (Kim et al., 2012; Laguna, Goldstein, Allen, Braun, \& Enguídanos, 2012).

\section{Intervention}

The introduction phrase was June through mid-August 2018. Flyers and posters of the utilization of a PC trigger for early PC consultation were placed in the oncology medicine nurse station and workroom. Nurse education is provided in a monthly staff meeting, during the huddle, and by email to the nurses. The PC trigger list is also introduced to the floor hospitalists by email.

The action phrase took place between August 23 and November 30, 2018. The primary nurses evaluated the patient's unmet needs according to the PC trigger and discussed opportunities for activating PC consultation during daily rounds with the internal medicine physician. Patients meeting at least one indicator were eligible for PC consultation per the protocol. The internal medicine physician acknowledged the proposal from the nurse and reviewed the triggers and patient condition before placing the consultation order. When PC consulting team received the electronic PC consultation order, the team visited the patient and assisted the hospitalists for symptom management and the goal of care discussion along with the subsequent follow-up visits during the hospital stay. Resources from PC team social worker also assisted family meeting set up, contact hospice facility, referral process, and family resource support; case managers also assisted with the discharge plan. 
The project leader also assisted the primary nurse in clarifying the PC trigger protocol questions and concerns in promoting the early recognition eligible patient for PC consultation. Day shift charge nurses and clinical nurse specialists also played the role of project resource for the PC trigger in communicating and collaborating between the hospitalists, the nurses, and the PC consulting team

Data source. Post-intervention data are collected from the UCSD electronic medical record, including palliative care consultation outcome of the symptom (e.g., pain, nausea/vomiting, anxiety, delirium), patient admission diagnosis, admission date, code status, first PC encounter date, discharge date, code status, and discharge destination.

Outcome. The primary outcome is time to the PC consult. The waiting time is measured from the time a patient was admitted to the oncology medicine floor to the time of the first encounter with the PC team at the bedside. The secondary outcomes are the changes of pain intensity after the consultation, days from consult to discharge alive, length of stay, the frequency of code status change, and consult rate.

Data Analysis. Descriptive statistics such as mean, median, and the difference will be chosen to summarize the project's performance because the advantages of user-friendly calculation formulas, it is easy for audiences to interpret the impact of the project. Pre- and postvalues will demonstrate the comparison of the time to consult, palliative care consult rate, pain intensity, number of changes in code status, and length of stay.

\section{Results}

\section{Patient Clinical Characteristics}

A total of 32 patients with metastatic cancer age 22 to 78 were seen by the PC consulting team per the triggers out of the 159-total admissions from August 23 to November 28, 2018, with 
more females than men $(59.3 \%$ vs. $40.6 \%)$. As Table 1 shows, the primary cancer site ranked top in lung (5) and blood (5), followed by breast (4), colon (3), testicles (2), appendix (2), bone (1), cervix (1), bile duct (1), liver (1), pancreas (1), esophageal (1), kidney(1), brain (1), skin (1), peripheral nerve sheet (1), and spindle cell (1). These advanced cancer involved at least one to most of five organs metastases. Among these group, 14 (43.7\%) patients passed during hospital stay including two signed up for hospice general inpatient care (GIP), four (12.5\%) discharged to home hospice, three (9\%) discharged to home with PC outpatient follow-up, two (6\%) remained in hospital stay, and the remaining nine $(28.1 \%)$ patients had a normal discharge to their home during the implementation period (see Figure 8).

\section{Time of Admission to Palliative Care Consult}

The post-implementation data resulted in a significant 2.29-day reduction in the time from admission to PC consult when compared the pre-data of average time to consult (2.87 days vs. 5.16 days). The significant of $55 \%$ less waiting period to PC consult implied the utilization of a comprehensive trigger tool guides an immediate medical-decision making for nurses and physicians when discussing the concerns of initiating PC referral. Moreover, as Hui and Bruera (2016) had mentioned, daily screening the patient condition by using the trigger tool has a benefit of preventing the professionals from missing high-risk potential candidates (see Figure 3).

\section{Pain Intensity}

A total of 30 patients consulted for advanced pain management during this period. We collected the pre-intervention data as patient-self reported on the NSR scale by the time the PC service evaluated the patient at the bedside. The post-intervention pain scale is collected at 24 hours, 48 hours, and 72 hours after the intervention from the electronic medical record; 
moreover, we also collected pain scale data by time for patients who were discharged alive (Figure 4).

In evaluating the improvement of pain intensity, we measured the difference between the pre and post data by subtracting the two measures. In interpreting the range of the improvement, we calculated the difference from the pre and post data into a percentage. Overall, the 30 patients enrolled in the PC program showed a promising pain management result. Most of the patients reported severe pain at the first-time encounter with the PC service at the bedside $(M=7.27$, $M d n=8)$; pain intensity subsided by least a $30 \% 24$ hours after the PC specialist changed the patient's pharmacology pain management regimen $(M=4.86, M d n=6)$. Progressively, pain intensity continued to decrease at a mean of $3.83(M d n=4)$ by 72 hours after the intervention. By the time when the patients discharge, the pain intensity had met $50 \%$ less to the time of admission.

\section{Length of Stay}

The length of stay had a significant decrease when comparing to pre-data. Mean length of stay including patients discharged alive, and those who died in the hospital, demonstrated a 3.5day reduction in stay (12.44 days vs. 15.94 days), and the mean length of stay for patients discharged alive was 4.42 days shorter (6.5 days vs. 10.92 days), respectively. Additionally, a patient discharged home alive reduced their stay 2.99 days post-intervention from the time of consult (5 days vs. 7.99 days) (see Figures 5 and 6).

\section{Code Status Change}

A total of 32 patients with advanced cancer were endorsed full code when arriving on the oncology medicine floor, which indicated the agreement of receiving chest compression and endotracheal tube intubation during the life-threatening situations in the hospital stay. 
Significantly, post-data revealed a more than $50 \%$ change in the code status after having a goal of care discussion with the PC service. Twelve patients transitioned to comfort care (43\%); five patients changed to do not resuscitate $(15 \%)$, and the rest of $40 \%(n=13)$ remained a full code when discharge (see Figure 7).

\section{Palliative Care Consult Rate}

The consulting rate was slightly lower (20\% vs. $23 \%$ ) than the pre-data. Given the predata had collected a year-long data sample, in turn, the data of consult rate is relatively more extensive than a 3-month long post- data ( pre 119/402 v.s. post 32/159).

\section{Discussion}

This EBP pilot study was designed to use a PC consultation trigger protocol to facilitate the PC consult process, optimize cancer-related symptoms intensity, and promote the goal of care discussion initiation in patients with metastatic cancer admitted to this floor promptly.

Measuring waiting time from patient admission to initial PC encounter at bedside was primarily intended to improve in the setting of patients were critically ill in their end of life. A significant $55 \%$ shorter waiting interval of 2.87 days reveals a standardized PC consult checklist played a crucial role in leading effective communication between the nurses and the physicians and urged the consultation happen in a short time interval. Though limited studies in early PC consultation by trigger model exist, a similar emergency room study also resulted in shorter waiting time to PC consultation and related to this pilot study (Kistler et al., 2015). Moreover, the standard referral criteria also avoided the confusion when examing patient's unmet needs; it improves the decision-making when meeting the potential candidates for PC consult. As a result, the consultation process is more effective and productive. Especially, when using the trigger 
screening with daily patient assessment as Hui and Bruera's (2016) recommendation, it lessened the chance of fail to recognize potential PC candidates.

Next, the secondary outcomes correspondingly showed definite improvements in pain intensity, length of stay from PC consult to discharge alive, the average length of stay, and the frequency of code status change followed by PC consultation, which correlated to the evidence of the literature research.

Pain management in cancer patients has been known to be very challenging; insufficient pain control affects patient's self-care, daily activities, and social life in many aspects (Ferrell, Sun, \& Hurria, 2015; Laguna et al., 2012; Zimmermann et al., 2014). Near 93\% $(n=30)$ the eligible patients were identified as the need for intensive pain management during the intervention period. A remarkable decrease of pain intensity by $33 \%$ in the first 24 hours after being seen by PC service, following by a significant $44-50 \%$ decrease of pain in day three that enables patients to resume their daily function in ambulating, self-care, and maintain their social life sooner and resulting in a shorter hospital stay. This applaudable outcome that enhances the benefit of the partnership of the hospitalists and PC team in taking care of cancer patients can lead to satisfied symptom management in an acute crisis

This study reflects the timing of the goal of care discussion in inpatient setting also determines the length of stay (LOS). When patients were identified that have a poor prognosis, the social worker arranged the family meeting consist of a hematologist, PC service and the primary team in discussing the options of care and support with the patient and family instead of continuing aggressive treatment at the end of life care. Once the patient and family decided on their preferred care, the social worker and case manager will assist the discharge plan in transition to home health, skilled nursing facility, or hospice referral. As a result, a significant 4- 
day reduction in the mean LOS including patients discharged alive and transitioned to comfort care was observed during the intervention period (see Figure 5). Also, the hospital stay of patient discharge alive and the period from the first PC visit at the bedside to discharge are trending down to 3.9 days shorter and 2.99 days shorter, respectively (see Figure 6).

PC service is well-known for assisting patient and family in understanding the disease trajectory and offering alternative treatment options through the disease course (LeBlanc \& ElJawahri, 2015). Impact of the goal of care conducted by PC service, significantly, total of 59\% patients $(n=19)$ changed their code status to comfort care $(n=14)$ and Do Not Resuscitate(no intubation, no chest compression; $n=5)$. Among these 14 cancer patients who changed to comfort care had distant metastases in one to five organs, most of them had passed away by a mean period of four days after the code status changed, the age of the patient range from $22-78$, and the average age is $48(M d n=52)$, men are more than female (57\% vs. 42\%). Moreover, among these 14 patients, $37 \%$ of patients' $(n=12)$ conditions were too sick and died in a short period after the admission, resulted in a lack of enough time to initiate the hospice referral after PC consultation. As a result, the loss of the opportunity for hospice referral also indirectly affected the total number of hospice utilization $(18 \%)$.

Advance care planning(ACP) has been introduced by the American Society of Clinical Oncology (ASCO) and NCCN since 2016 for standard oncologic patient care whereas the ACP conversation with patients in oncology clinic is less than $10 \%$ (Bestvina \& Polite, 2017). Similarly, all of 32 patients with advanced cancer found maintained full code when admitted to the hospital; most of these patients are at the very end of their life. A lack of fully understand their disease prognosis can take roughly two weeks from the first encounter with the PC service to change to comfort care depending on the patient and family's perceptions and acceptance to 
the prognosis. There is no available information to answer the question of the absence of advance care planning before the admission, but this study offers a lesson for providers that an early goal of care discussion should start sooner when the disease progresses.

Last, postintervention consulting rates are slightly lower compared to preintervention data from March 2017 to April 2018 (post 20\% vs. pre 24\%). When evaluating the performance quality of the project, considering the underlying factors is imperative. The preintervention data sample size is relatively more significant than the 3-month intervention data sample size, and the total admission samples are not complete cancer diagnoses during the project implementation phase. Consequently, the comparison has limited impact on the consulting rate by an early PC consultation activation. Hence, the consulting rate may require a more extended continuing observation period to produce convincing results. Even though this consulting rate overall presents a higher PC referral rate when comparing the consulting rate from 2017 National Palliative Care Registry step-down unit (14.1\%) and oncology floor, a more extended intervention period would validate a reasonable comparison.

\section{Implication and Conclusion}

This project represents valuable impacts on patient outcomes. Also, it enhanced interprofessional communication. When the physicians and nurses can identify the high-risk candidate earlier, the degree of symptom intensity reduced in a shorter period after appropriate management; and an immediate goal of care discussion was able to happen for a patient who is in need. Knowing using a trigger for PC has various advantages, the number of using a triggerbased model for PC consultation is increasing across the nation, of a total $34.6 \%$ hospital palliative care program are found to have their standard trigger tool to meet patient's needs ( National Palliative Care Registry, 2017). Hence, this project also demonstrated an example of 
implementing a trigger tool for PC consult in an inpatient setting for other departments which pursuit for similar project development.

Aside from the above positive patient outcome, this study found that a high proportion of patients needed advance directive completion. Low ACP initiative sequentially leads patient and family unable to understand their condition entirely and unable to consider other alternative options before the condition becomes imminent life-threatening(Adelson et al., 2017). Consequently, it added more challenges and stress for medical staff, patient, and family in many aspects when facing a patient in a rapidly deteriorating. Barriers of the low ACP utilization including the lack of time, training, and inadequately billing submission had been mentioned in a study ( Bestvina \& Polite, 2017). Improving this problem, suggestions of providing the education, introducing proper billing instruction and teamwork project may proceed to a success ( Bestvina \& Polite, 2017; Jones, Acevedo, Bull, \& Kamal, 2016).

Last, given the patients who admitted to this floor had poor disease prognosis, the need for advanced directive initiation is imperative. A suggestion of adding advanced directive screening in addition to the trigger for PC consultation in the future study may apply much earlier goal of care initiative and may able to meet an appropriate outpatient PC utilization and hospice care referral. 


\section{References}

American Cancer Society. (2017). Cancer facts \& figures 2017. Retrieved from https://www.cancer.org/content/dam/cancer-org/research/cancer-facts-and$\underline{\text { statistics/annual-cancer-facts-and-figures/2017/cancer-facts-and-figures-2017.pdf }}$

Adelson, K., Paris, J., Horton, J. R., Hernandez-Tellez, L., Ricks, D., Morrison, R. S., \& Smith, C. B. (2017). Standardized criteria for palliative care consultation on a solid tumor oncology service reduces downstream health care use. Journal of Oncology Practice, 13, e431-e440. doi:10.1200/JOP.2016.016808

Bakitas, M. A., Tosteson, T. D., \& Li, Z. (2015). Early versus delayed initiation of concurrent palliative oncology care: Patient outcomes in the Enable III randomized controlled trial. J Clin Oncol, 33(13) 1438-1445. doi:10.1200/JCO.2014.58.6362

Bestvina, C. M., \& Polite, B. N. (2017). Implementation of Advance Care Planning in Oncology: A Review of the Literature. Journal of Oncology Practice, 13(10), 657-662. https://doi.org/10.1200/JOP.2017.021246

Buckwalter K. C., Cullen, L., Hanrahan, K., Kleiber, C., McCarthy A. M., . . Tucker, S. (2017). Iowa model of evidence-based practice: Revisions and validation. Worldviews on Evidence-Based Nursing, 14, 175-182. doi:10.1111/wvn.12223

Creutzfeldt, C. J., Wunsch, H., Curtis, J. R., \& Hua, M. (2015). Prevalence and outcomes of patients meeting palliative care consultation triggers in neurological intensive care units. Neurocritical Care, 23(1), 14-21. doi:10.1007/s12028-015-0143-8

Institute of Medicine (IOM). (2015). Dying in America: Improving quality and honoring individual preferences near the end of life. Washington, DC: National Academies Press. 
Ferrell, B., Sun, V., \& Hurria, A. (2015). Interdisciplinary palliative care for patients with lung cancer. Journal of Pain Symptom Management, 50. doi:10.1016/j.jpainsymman.2015.07.005

Ferrell, B. R., Temel, J. S., Temin, S., Alesi, E. R., Balboni, T. A., Basch, E. M., ... Smith, T. J. (2016). Integration of palliative care into standard oncology care: American Society of Clinical Oncology clinical practice guideline update. Journal of Clinical Oncology, 35, 96-112. doi:10.1200/JCO.2016.70.1474

Groenvold, M., Petersen, M. A., Damkier, A., Neergaard, M. A., Nielsen, J. B., Pedersen, L., ... Johnsen, A. T. (2017). Randomised clinical trial of early specialist palliative care plus standard care versus standard care alone in patients with advanced cancer: The Danish Palliative Care Trial. Palliative Medicine, 31, 814-824. doi:10.1177/0269216317705100

Hua, M. S., Li, G., Blinderman, C. D., \& Wunsch, H. (2014). Estimates of the need for palliative care consultation across united states intensive care units using a trigger-based model. American Journal of Respiratory and Critical Care Medicine, 189, 428-436. doi:10.1164/rccm.201307-1229OC

Hui, D., \& Bruera, E. (2016). Integrating palliative care into the trajectory of cancer care. Nature Reviews. Clinical Oncology, 13, 159-171. doi:10.1038/nrclinonc.2015.201

Jones, C. A., Acevedo, J., Bull, J., \& Kamal, A. H. (2016). Top 10 Tips for Using Advance Care Planning Codes in Palliative Medicine and Beyond. Journal of palliative medicine, 19(12), 1249-1253.

Kim, E. B., Han, H.-S., Chung, J. H., Park, B. R., Lim, S., Yim, K. H., ... Kim, S. T. (2012). The effectiveness of a self-reporting bedside pain assessment tool for oncology inpatients. Journal of Palliative Medicine, 15, 1222-1233. doi:10.1089/jpm.2012.0183 
Kistler, E. A., Sean Morrison, R., Richardson, L. D., Ortiz, J. M., \& Grudzen, C. R. (2015). Emergency department-triggered palliative care in advanced cancer: proof of concept. Academic Emergency Medicine, 22, 237-239. doi:10.1111/acem.12573

Laguna, J., Goldstein, R., Allen, J., Braun, W., \& Enguídanos, S. (2012). Inpatient palliative care and patient pain: Pre- and post-outcomes. Journal of Pain and Symptom Management, 43, 1051-1059. doi:10.1016/j.jpainsymman.2011.06.023

LeBlanc, T. W., \& El-Jawahri, A. (2015). When and why should patients with hematologic malignancies see a palliative care specialist? ASH Education Program Book, 2015, 471478. doi:10.1182/asheducation-2015.1.471

Levy, M., Smith, T., Alvarez-Perez, A., Back, A., Baker, J. N., Beck, A. C., ... Scavone, J. L. (2016). Palliative Care Version 1.2016. Journal of the National Comprehensive Cancer Network, 14(1), 82-113. https://doi.org/10.6004/jnccn.2016.0009

Melnyk, B. M., \& Fineout-Overholt, E. (2015). Evidence-based practice in nursing and healthcare: A guide to best practice. Philadelphia, PA: Wolters Kluwer.

National Palliative Care Registry. (2017). 2017 data summary (non-pediatric palliative care programs). Retrieved from https://registry.capc.org/wp-content/uploads/2018/07/2017Data-Summary.pdf

Parikh, R. B., Kirch, R. A., Smith, T. J., \& Temel, J. S. (2013). Early specialty palliative care Translating data in oncology into practice. New England Journal of Medicine, 369, 23472351. doi:10.1056/NEJMsb1305469

Reuben, D. B., Mor, V., \& Hiris, J. (1988). Clinical symptoms and length of survival in patients with terminal cancer. Archives of Internal Medicine, 148, 1586-1591. doi:10.1001/archinte.1988.00380070082020 
Wentlandt, K., Krzyzanowska, M. K., Swami, N., Rodin, G. M., Le, L. W., \& Zimmermann, C. (2012). Referral practices of oncologists to specialized palliative care. Journal of Clinical Oncology, 30, 4380-4386. doi:10.1200/JCO.2012.44.0248

WHO Definition of Palliative Care http://www.who.int/cancer/palliative/definition/en/. Accessed

Zimmermann, C., Swami, N., Krzyzanowska, M., Hannon, B., Leighl, N., Oza, A., ... Lo, C. (2014). Early palliative care for patients with advanced cancer: A cluster-randomized controlled trial. The Lancet, 383(9930), 1721-1730. doi:10.1016/S0140-6736(13)624162 
Figure 1.

Oncology Medicine Floor Palliative Care Triggers

Admission date

Diagnosis

1.Request from patient/Family/Caregiver

Yes or No

2. Need for clarification of treatment goals.

Yes or No

3. Severe Constipation that does not respond

Yes or No

to current bowel regimen

4. Delirium

Yes or No

5. Dyspnea

Yes or No

6. Sever Pain that does not respond to

Yes or No

current pain management regimen

7. Refractory Nausea/vomiting that does not respond to current treatment

Yes or No

\section{Total Yes}

When a Patient has $\geq 1$ of the above indications will be eligible for PC consultation. Please notify the primary care providers the screening result for consultation initiation.

The above PC consultation indications are adopted from National Comprehensive Cancer Network (NCCN) Clinical Practice Guidelines in Oncology. Palliative Care, 2016. http://www.nccn.org/professionals/physician_gls/pdf/palliative.pdf. 
Figure 2 .

\section{Establishment of UCSD TICU/MICU/CVICU Palliative Care Triggers}

1. Foreseeable decrease in quality of life after discharge

2. Family request

3. Futility declared or considered by primary team/RN

4. Death expected within ICU stay

5. ICU stay $>10$ days

6. A diagnosis with median survival $<6$ months

7. A patient with advanced malignancy

8. Permanent severe cognitive impairment

9. Progressive multi-organ system failure not responding to aggressive care

10. More than one hospital admission for the same diagnosis in the last 30 days

11. Two or more unscheduled ICU admissions within the same hospitalization*

12. Conflict in goals of care (between interdisciplinary teams or family)

13. Poorly controlled symptoms despite current treatment

14. A general sense that a palliative care consult is needed 
Table 1

Patient Demographics

\begin{tabular}{lll}
\hline Demographics & $\mathrm{n}$ & $\%$ \\
\hline Age, y & 3 & 9.3 \\
$20-30$ & 6 & 18.7 \\
$31-40$ & 4 & 12 \\
$41-50$ & 8 & 25 \\
$51-60$ & 8 & 25 \\
$61-70$ & 3 & 9.3 \\
$71-80$ & & \\
Gender & 13 & 40.6 \\
Male & 19 & 59.3 \\
Female & & \\
Cancer Diagnosis & 1 & 3 \\
Brain & 4 & 12 \\
Breast & 1 & 3 \\
Esophagus & 5 & 15 \\
Lung & 1 & 3 \\
Pancreas & 1 & 3 \\
Liver & 1 & 3 \\
Bile duct & 3 & 9.3 \\
Colon & 2 & 6 \\
Appendix & 1 & 3 \\
Kidney & 2 & 6 \\
Testicles & 1 & 3 \\
Cervix & 1 & 3 \\
Skin & 1 & 3 \\
Bone & 1 & 3 \\
Peripheral never sheet & 1 & 3 \\
Spindle cell & 5 & 15 \\
Haematology & & \\
\hline & & \\
\hline
\end{tabular}


Figure 3. Admission to Initial Consult

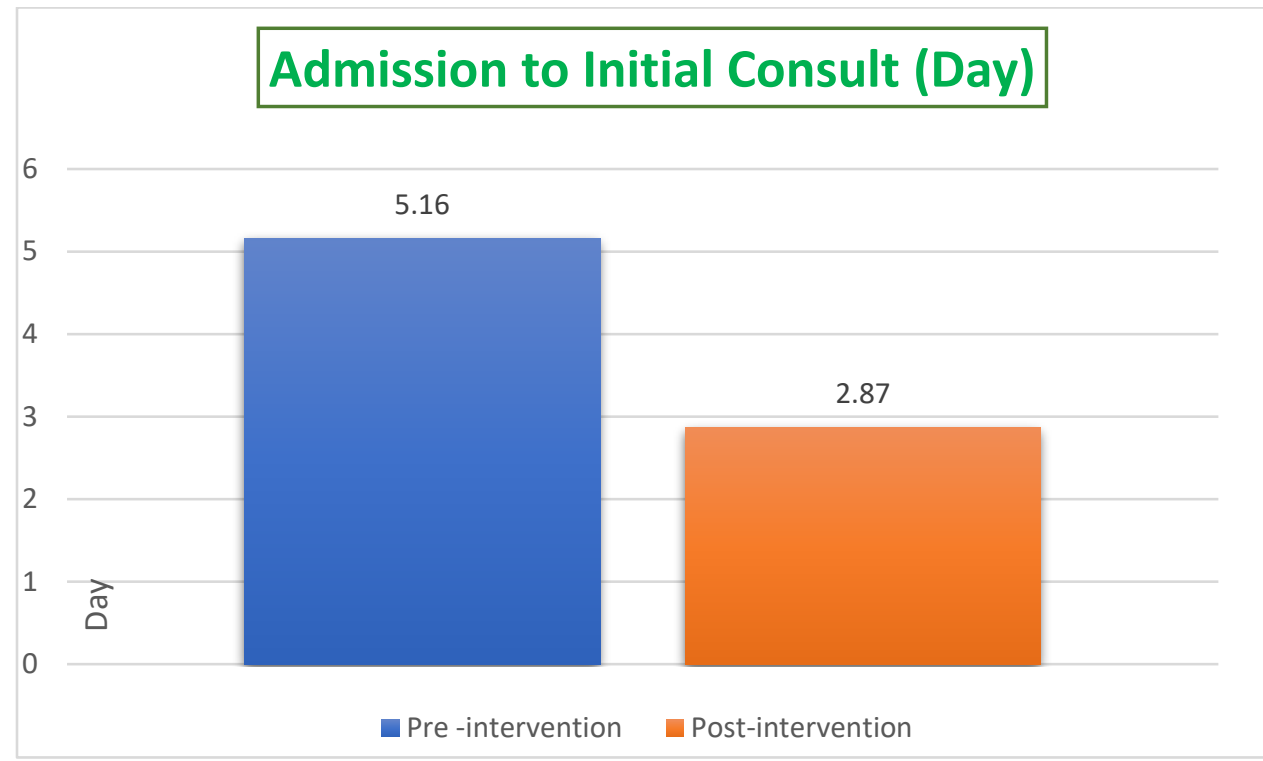

Figure 3. The comparison of pre and post data of the mean waiting time from the patients who were admitted to the medical oncology floor to the time having the first encounter with palliative care service at the bedside. 
Figure 4. Pain Intensity

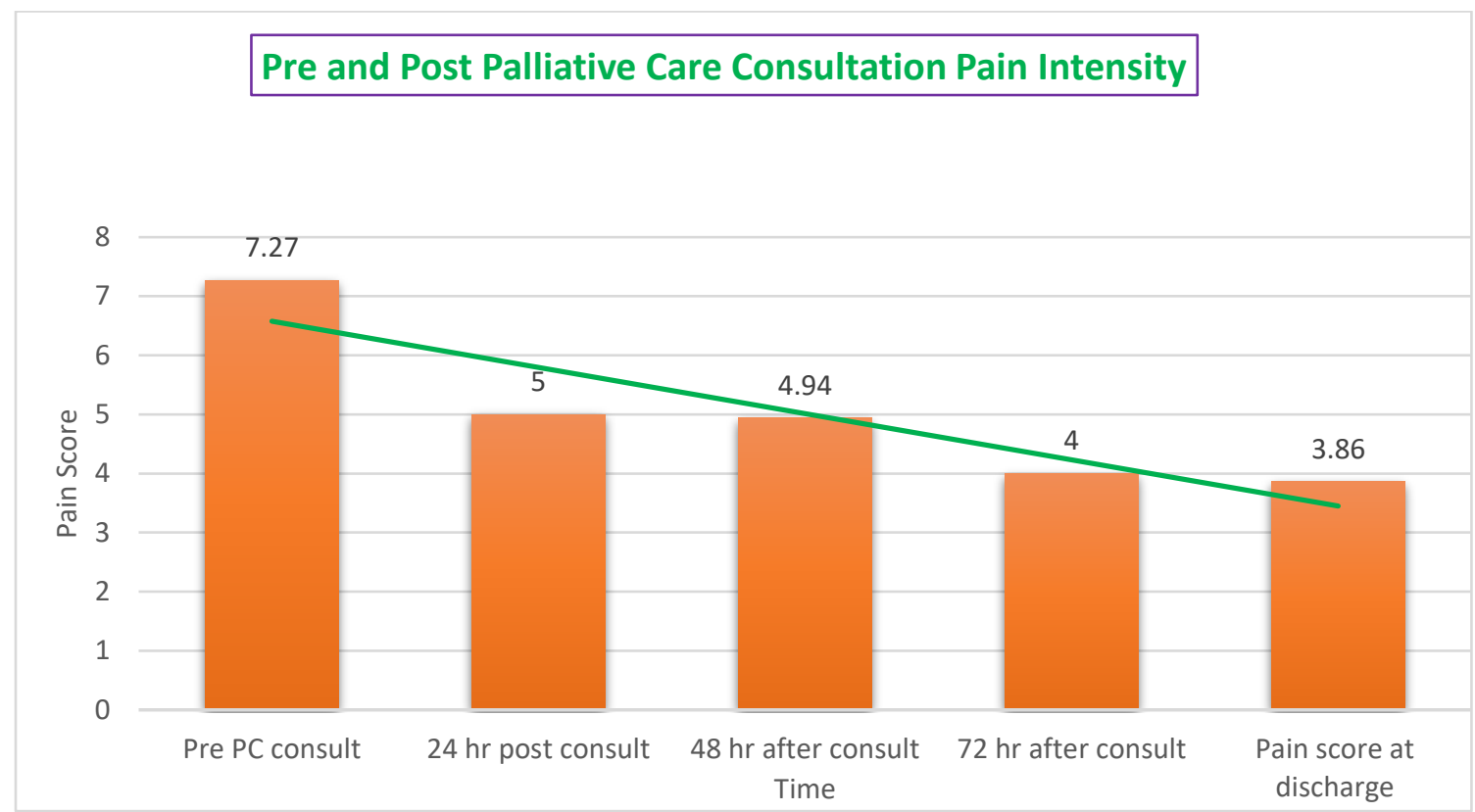

Figure 4. The graph illustrates the changes in pain intensity before and after receiving palliative care consultation. 
Figure 5. Average Length of Stay Included Comfort Care and GIP

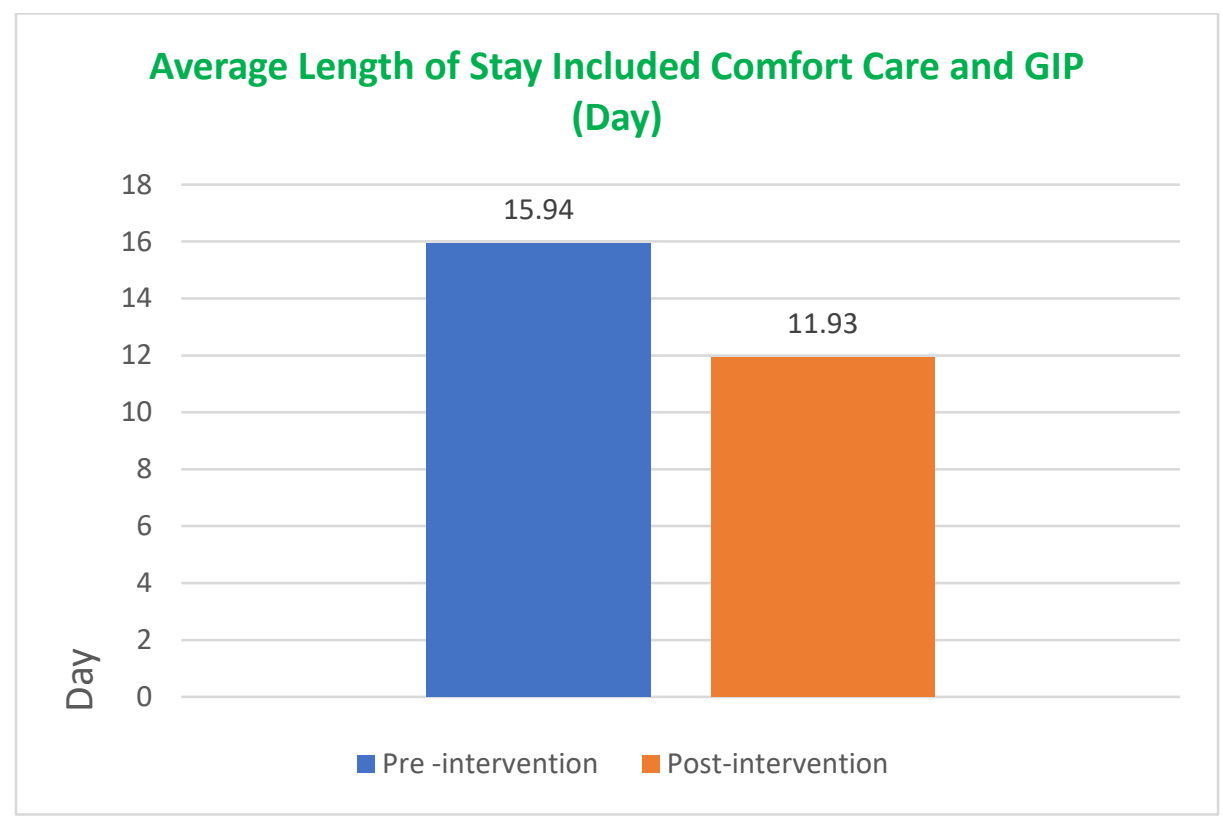

Figure 5. This bar chart represents the average length of stay including patient discharge alive and died during the hospital stay before and after the project implementation. 
Figure 6. Average Length of Stay Discharge Alive

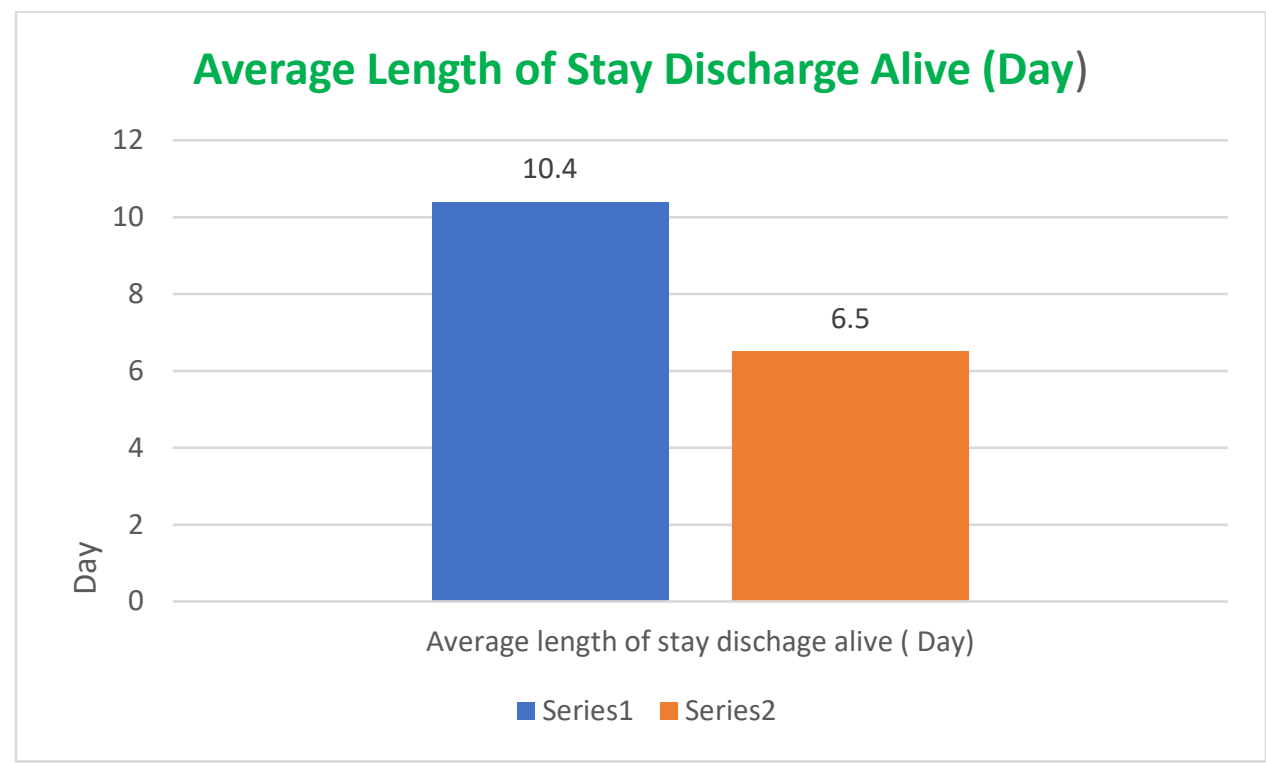

Figure 6. This bar chart represents the comparison of before and after the trigger-based model palliative care consultation on the average length of stay of patients who were discharged alive. 
Figure 7. Percentage of Code Status Change Post PC Consultation

\section{PERCENTAGE OF CODE STATUS CHANGE POST PC CONSULTATION}

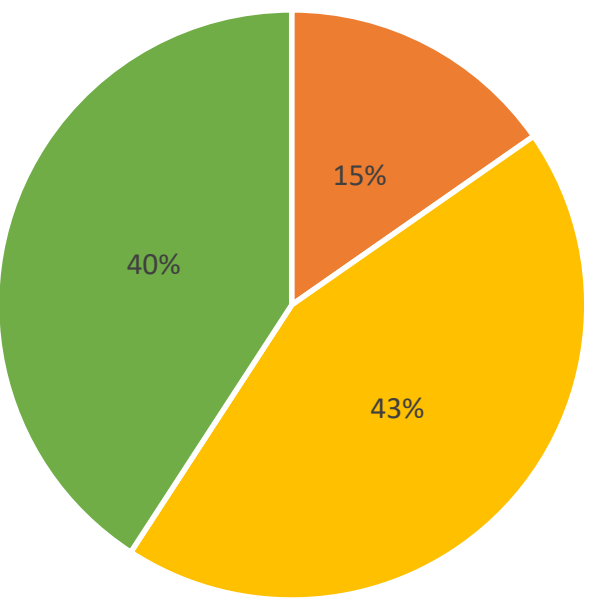

- Full to DNAR FULL care $\quad$ - Changed to Comfort Care $\quad$ - Remain Full Code

Figure 7. This pie chart represents the percentage of patients who were admitted to medical oncology floor with code status of full code during the project intervention period, and they changed to either Do not resuscitate or Comfort Care after receiving palliative care consultation. 
Figure 8. Number of Discharge Disposition After Palliative Care Consultation

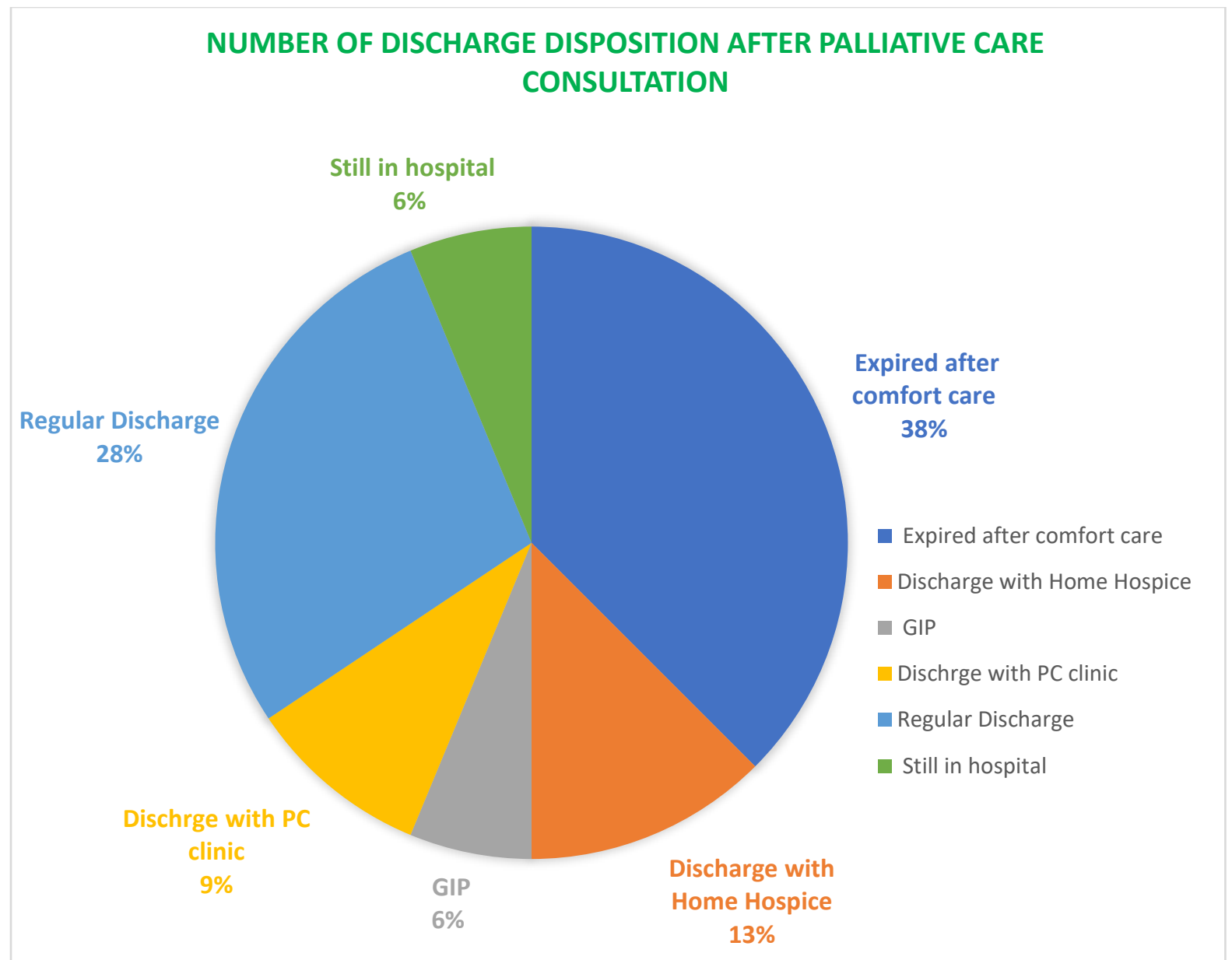

Figure 8 . The pie chart represents the percentage of patient discharge disposition after the patients who received the palliative care consultation and goal of care discussion during the intervention period. 